\title{
SEMA4D Knockdown Attenuates $\beta$-Catenin-Dependent Tumor Progression in Colorectal Cancer
}

\author{
Mahsa Rezaeepoor $\left(\mathbb{D},{ }^{1}\right.$ Golnaz Rashidi ${ }^{D},{ }^{1}$ Mona Pourjafar $\left(\mathbb{D},{ }^{2}\right.$ Chiman Mohammadi (D), \\ Ghasem Solgi $\left(\mathbb{D},{ }^{1}\right.$ and Rezvan Najafi $\mathbb{i}^{2}$ \\ ${ }^{1}$ Department of Immunology, Faculty of Medicine, Hamadan University of Medical Sciences, Hamadan, Iran \\ ${ }^{2}$ Research Center for Molecular Medicine, Hamadan University of Medical Sciences, Hamadan, Iran \\ Correspondence should be addressed to Rezvan Najafi; najafi2535@gmail.com
}

Received 14 April 2021; Revised 14 June 2021; Accepted 8 July 2021; Published 22 July 2021

Academic Editor: Somchai Chutipongtanate

Copyright (C) 2021 Mahsa Rezaeepoor et al. This is an open access article distributed under the Creative Commons Attribution License, which permits unrestricted use, distribution, and reproduction in any medium, provided the original work is properly cited.

\begin{abstract}
Semaphorin 4D (SEMA4D), a protein originally demonstrated to regulate the immune system and axonal growth cone collapse in the developing central nervous system, is overexpressed in various human malignancies, including colorectal cancer (CRC). This investigation was undertaken to examine the effects of SEMA4D silencing on the biological properties of the CRC cell line. SW48 cells were transfected with a siRNA-targeting SEMA4D. The mRNA expression of underlying pro- and antiapoptotic proteins including Bax, Bcl-2, P53, and caspase-3, cancer stem cell (CSC) markers, epithelialmesenchymal transition (EMT) markers, MMP-2, and MMP-9 was examined using qRT-PCR. Further, the protein expression of E-cadherin and $\beta$-catenin was confirmed by Western blot. SW48 cell migration and MMP activity were detected using scratch and zymography analysis, respectively. Finally, the apoptosis rate was assessed via the flowcytometry test. SEMA4D knock-down was associated with a considerable suppression of in vitro cell viability, EMT-related genes, CSC markers, $\beta$-catenin signaling pathway, sphere-forming, cell migration, and MMP-2 activity as well as induction of apoptosis. This study identifies the inhibitory effects of SEMA4D gene silencing on tumor progression. Thereby, this might conclude a possible alternative to cancer therapy by targeting several prominent pathways involved in cancer through SEMA4D suppression.
\end{abstract}

\section{Introduction}

Colorectal cancer (CRC) is the third most prevalent malignancy as well as the second major cause of cancer-related mortality all over the world [1]. Several therapeutic modalities have been proposed for CRC based on pathological characteristics of the tumor such as surgery, radiotherapy, and chemotherapy [2]. Recurrence, metastasis, and resistance to treatment have caused problems in the therapy of CRC [3]. Tumors consist of several kinds of cancer cells contributing to the heterogeneity of the tumor. Several investigations have indicated that a number of malignant cells like colon cancer cells acquire the features of cancer stem cells (CSCs) via epithelial-mesenchymal transition (EMT). CSCs play a crucial role in the initiation and progression of cancer $[4,5]$. Brabletz et al. have proved the presence of two stem cell subpopulations in colon cancer. The first was able to initiate the tumor and the second could spread the tumor-forming metastasis. Hence, the CSC theory has been presented as a novel perspective to explore tumor initiation, recurrence, and metastasis [6].

Epithelial-mesenchymal transition (EMT) is a process by which cancer cells attain an invasive phenotype leading to metastasis. During the progression of tumor, EMT contributes remarkably to the malignant features of tumors, including local invasion and distant metastasis. EMT has recently been recognized as a key phenomenon that tightly adjusts the CSCs [7]. During the EMT process, the transformed epithelial cells acquire mesenchymal traits that convert them to metastatic cells. A hallmark of metastatic cells is the lost expression of epithelial markers such as E-cadherin as well as induction of mesenchymal markers including $\mathrm{N}$-cadherin, 
vimentin, and Zeb1 [8]. Several signaling pathways, including hepatocyte growth factor (HGF), epidermal growth factor (EGF), transforming growth factor beta (TGF- $\beta$ ), Notch, and $\mathrm{Wnt} / \beta$-catenin, regulate EMT, and the activation of $\mathrm{Wnt} / \beta$-catenin pathway is commonly seen in many malignant tumors $[9,10]$.

Wnt is a vital signaling pathway associated with selfrenewal of tissues and homeostasis. $\beta$-Catenin is a prominent mediator of Wnt signaling pathway, leading to signal transmission from the cytoplasm through the nucleus [11]. Activation of $\mathrm{WNT} / \beta$-catenin signaling can result in $\beta$-catenin accumulation in the nucleus, which is accompanied with a poor prognosis among CRC patients [12]. Recent findings have evidently suggested the critical effect of $\mathrm{WNT} / \beta$-catenin dysregulation on the induction of EMT process as well as sustained CSC expansion in the pathogenesis of CRC $[11,13]$.

The semaphorins are glycoproteins discovered in 1990s and initially described as axon guidance factors, which have later been implicated in the regulation of immune responses, angiogenesis, as well as pathogenic processes, including tumor inhibition and progression [14]. Semaphorin 4D (SEMA4D) was originally described in immune cells as CD100 antigen and was the first semaphorin family member found to possess immunoregulatory activities. SEMA4D is synthesized as a transmembrane molecule, the soluble extracellular portion of which can be shed following metalloprotease-mediated cleavage. Notably, SEMA4D has three known receptors, namely, the high, intermediate, and low affinity PlexinB1, PlexinB2, and CD72, respectively, which are mainly expressed by immune cells [15]. Plexin$\mathrm{B} 1$ is expressed on vascular endothelial cells (VECs) at high levels, inducing angiogenesis by an independent mechanism apart from other angiogenic promoters such as vascular endothelial growth factor (VEGF-a), basic fibroblast growth factor (bFGF), and HGF [16]. Upregulation of Plexin-B1 is demonstrated on breast, pancreatic, and colorectal carcinoma tissues [17-19]. As an immune semaphorin, SEMA4D is able to regulate tumor microenvironment by differentiating monocytes toward tumor-supportive macrophage phenotype, namely, M2 macrophages with high expression of CD163 [20]. Moreover, SEMA4D is elevated in a variety of tumor tissues relative to normal tissue cells, including breast, cervical, epithelial ovarian, and prostate cancers as well as CRC $[18,21-24]$. It has been reported that the coexpression of SEMA4D and PlexinB1 is a risk factor for CRC relapse and that it may be an effective prognostic biomarker for recurrence of CRC [17]. Targeted therapy is an important emerging area for cancer treatment. Based on clinical applicability of semaphorins and their receptors as well as their prominent roles especially in the tumor progression, semaphorins have turned into compelling therapeutic targets, then we followed the methods of our previous study [25].

The goal of the present study was to determine the changes of tumor progression following the inhibition of SEMA4D expression using siRNA. The findings indicated that targeting SEMA4D for inhibition might serve as a therapeutics solution for the suppression of invasion, migration, and viability of SW48 cell line, which is related to CRC.

\section{Materials and Methods}

2.1. Cell Culture. As a model of colon cancer cells, the SW48 human colorectal cancer cell line, purchased from the National Cell Bank of Pasteur Institute (Tehran, Iran), was used. The cells were cultured in Dulbecco's modified Eagle's medium supplemented with $10 \%$ heat-inactivated fetal bovine serum (FBS; Gibco, Waltham, MA) and 1\% penicillin or streptomycin (Gibco), then were incubated in a humidified incubator with a temperature of $37^{\circ} \mathrm{C}$ and $5 \% \mathrm{CO} 2$ for $72 \mathrm{~h}$.

2.2. Cell Transfection. SEMA4D siRNA was purchased from Bioneer (South Korea) and cells at about $60-70 \%$ confluence were transfected with siRNA $(30 \mathrm{nmol})$ using HiPerfect Transfection Reagent (Qiagen, USA). SW48 cells were also transfected with scrambled siRNA as it does not target any known mammalian gene. Briefly, $10^{5}$ cells were seeded (per well) in a 12-well plate. For each transfection sample, a complex was prepared: the siRNA was diluted in $100 \mu \mathrm{l}$ of OptiMEM medium (Gibco) and $9 \mu$ l of HiPerfect. The complex was blended gently and was incubated $15 \mathrm{~min}$ at room temperature. Before adding the complex, the culture medium was renewed (without FBS and Pen/strep). Then, transfection complex was added to each well, containing cells and medium, and mixed softly by rocking the plate back and forth. Eventually, the plate was incubated at $37^{\circ} \mathrm{C}$ in a humidified incubator with $5 \% \mathrm{CO} 2$ for $72 \mathrm{~h}$.

2.3. Cell Viability Assay. To evaluate cell viability, the colorimetric MTT assay was used. Cells were seeded in 96-well plates at a density of 8000 cells in $50 \mu \mathrm{l}$ per well, and then, cells were treated with final concentrations of siRNA (30 nmol). After incubation for 24 hours, 3-(4, 5-Dimethylthiazol-2-yl)-2, 5-diphenyltetrazolium bromide (Sigma Aldrich, St Louis, MO, USA) was added and incubated for 4 hours at $37^{\circ} \mathrm{C}$. The medium was discarded, and then, $100 \mu$ DMSO (Sigma-Aldrich) was added. The optical density of solubilized Formazan was measured at $570 \mathrm{~nm}$ using an automatic microplate reader.

2.4. RNA Isolation and Quantitative Reverse-Transcription Polymerase Chain Reaction (RT-qPCR). Total RNA was isolated from the SW48 cell line using RNX-Plus Kit (Cinnagen, Tehran, Iran), $72 \mathrm{~h}$ after transfection, as recommended by the manufacturer. Purity and concentration of RNA were evaluated with NanoDrop, and total RNA was turned to cDNA with reverse transcriptase using a first-strand cDNA synthesis kit (Takara, Japan). Real-time PCR assay was conducted in triplicate using RealQ plus 2x master (Ampliqon, Denmark). The standard PCR conditions were as follows: $95^{\circ} \mathrm{C}$ for $15 \mathrm{~min}$ incubation, followed by 40 amplification cycles of denaturation at $95^{\circ} \mathrm{C}$ for $30 \mathrm{~s}$, annealing at $61^{\circ} \mathrm{C}$ for $30 \mathrm{~s}$ and extension $72^{\circ} \mathrm{C}$ for $30 \mathrm{~s}$ using a light cycler instrument (Roche 96 system, Germany). Primer sequences used for this study are listed in Table 1. Data were analyzed by normalizing with GAPDH as an internal control, and the relative expression of genes was plotted using the $2^{-\Delta \Delta \mathrm{Ct}}$ method. 
TABLE 1: Specific primer sequences for qRT-PCR.

\begin{tabular}{lcc}
\hline Gene & Sense strand & Antisense strand \\
\hline SEMA4D & AATGTTTGACGACACTGATGGT & TCTTTGCTGGTGCTAGAGATG \\
MMP2 & GGAGCATGGCGATGGATACC & TTCACACGGACCACTTGGC \\
MMP9 & GGTGATTGACGACGCCTTTG & AACCGAGTTGGAACCACGA \\
Bax & CCGCCGTGGACACAGACT & TTGAAGTTGCCGTCAGAAAACA \\
Bcl-2 & TGGAGAGTGCTGAAGATTGA & GTCTACTTCCTCTGTGATGTTGTAT \\
P53 & TAACAGTTCTGCATGGGCGGC & AGGACAGGCACAAACACGCACC \\
CD44 & AATGGTCGCTACAGCATCTC & GCCCTTCTATGAACCCATACC \\
CD133 & GAGTCGGAAACTGGCAGATAG & AACGCCTTGTCCTTGGTAG \\
BMI1 & CATCCACAGTTTCCTCACATTTC & GAAGTTGCTGATGACCCATTTAC \\
E-cadherin & CTTCACCTGACAGATCCAAGTC & CCTTCCATCCCTTCCTGTTTAG \\
N-cadherin & AGAACGCATTGCCACATACA & GAGGATGGTGTAAGCGATGG \\
Vimentin & ATTCGGGTAATCCTCCCAAATC & CCCACAATCCTGTCCACATC \\
ZEB1 & CATTGAGATTGCCACCTAC & CGTTGATAACCTGTCCATC \\
DCLK1 & ATTCGGGTAATCCTCCCAAATC & TTTCACTGTCTTCATCCTCTTCCC \\
GAPDH & TTGCTCCAGATCGTTAGAAGG & CAGGAAGGTCTCATTGAACAC \\
\hline
\end{tabular}

2.5. Western Blot Analysis. Total protein was isolated from harvested SW48 cells after $72 \mathrm{~h}$ using $0.2 \mathrm{ml}$ of radioimmuno precipitation assay (RIPA) buffer (Santa Cruz, USA), and concentration was measured by the Bradford protein assay. $100 \mu \mathrm{g}$ proteins with equal volumes were separated on $10 \%$ sodium dodecyl sulfate-polyacrylamide gel electrophoresis (SDS-PAGE) and then transferred from the gel onto a nitrocellulose membrane. After blocking, first antibodies including rabbit monoclonal anti-E-cadherin (ab40772, 1:7,000), rabbit monoclonal anti- $\beta$-catenin (ab32572, $1: 5000)$, and rabbit anti-beta-actin (ab8227, $1: 5,000)$ were incubated with the membrane at $4^{\circ} \mathrm{C}$ overnight. The secondary antibody (anti-rabbit antibody conjugated to horseradish peroxidase) was used, and bands were visualized using enhanced chemiluminescence detection kit. To quantify the intensity of bands, the Image J software was used.

2.6. Zymography. To evaluate the MMP-2/-9 enzymatic activities, zymography was performed. First, the supernatants of SW48 cell line (controls and transfected cells) were collected. Then, defined amounts of proteins were loaded in $10 \%(w / v)$ polyacrylamide gels containing $1 \mathrm{mg}$ gelatin $/ \mathrm{ml}$. Electrophoresis was run at $120 \mathrm{~V}$ for $2 \mathrm{~h}$. To renature the gel, it was immersed in $2.5 \%$ Triton X-100 buffer for $2 \mathrm{~h}$ and then incubated in developing buffer for $72 \mathrm{~h}$ at $37^{\circ} \mathrm{C}$ (50 mM Tris- $\mathrm{HCl}, 200 \mathrm{mM} \mathrm{NaCl}, 5 \mathrm{mM} \mathrm{CaCl} 2$, and $0.01 \%$ $\mathrm{NaN} 3, \mathrm{pH} 7.5)$. Finally, the gel was stained with $0.1 \%$ Coomassie brilliant blue G-250 in a $40 \%$ methanol and $10 \%$ acetic acid solution and then destained in $40 \%$ methanol, $10 \%$ acetic acid, and deionized water. Gel was scanned, and vivid bands were analyzed by NIH ImageJ software.

2.7. Flow Cytometry-Based Apoptosis Analysis. Apoptosis of the cells was measured according to the manufacturer's instructions, using an Annexin-V-FITC/Propidium Iodide (PI) kit (MabTag, Germany). Briefly, SW48 cell lines were collected after transfection. After washing and centrifuging at $300 \mathrm{~g}$ for $5 \mathrm{~min}$ at $4^{\circ} \mathrm{C}$, the cell pellet was resuspended in cold binding buffer. The Annexin V-FITC and PI solutions were added to the cell suspension and gently mixed. Following 15 min of incubation time, apoptosis and cell death were analyzed using Attune NxT acoustic focusing cytometer (Life technology, USA) and FlowJo software 10.

2.8. Colony-Forming Assay. To determine cell growth and survival, colony-formation assay was conducted [26]. 400 cells (per well) of transfected SW48 and control were seeded on a 6-well plate and allowed to grow for 10 days. The cell colonies were then stained with $5 \%$ crystal violet for $45 \mathrm{~min}$. After that, they were washed twice with PBS and distilled water and counted. To evaluate efficiency, the ratios of the visible colonies from all seeded cells were calculated. The survival fraction was clarified by the percentage of transfected cells compared to the control.

2.9. Sphere Formation Assay. After 72 h of transfection, SW 48 cells were seeded in a 6-well low attachment plate at a density of 30,000 cells per well with $20 \mathrm{ng} / \mathrm{ml}$ of epidermal growth factor (EGF) and $20 \mathrm{ng} / \mathrm{ml}$ of basic fibroblast growth factor (b-FGF). After 7 days of incubation at $37^{\circ} \mathrm{C}$, the number of formed spheroids $>50 \mu \mathrm{m}$ in diameter was counted using ImageJ software [27, 28].

2.10. Wound Healing Assay. Briefly, $10^{5}$ SW48 were seeded into 12 -well plates. $72 \mathrm{~h}$ after transfection, an incision was made on the cell layers with a sterile pipette tip $(100 \mu \mathrm{l})$. To examine the cell migration over the incision, an inverted microscope was used after $24-48 \mathrm{~h}$, and finally, distances in the scratch area were analyzed by the NIH Image J software.

2.11. Statistical Analysis. Evaluation of data analysis among different groups was performed by PRISM software using unpaired $T$-tests. Data were reported as the mean \pm SD and 


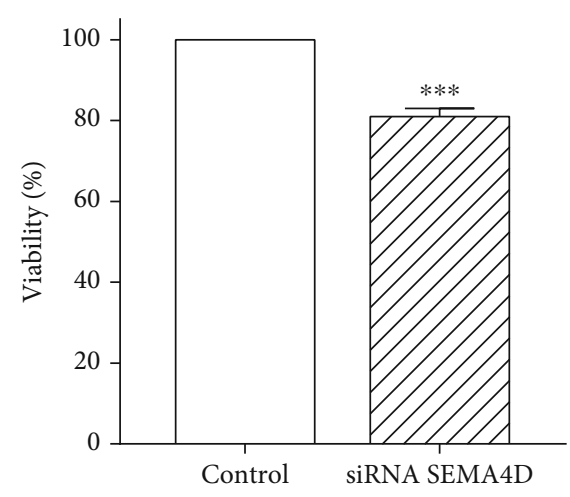

(a)

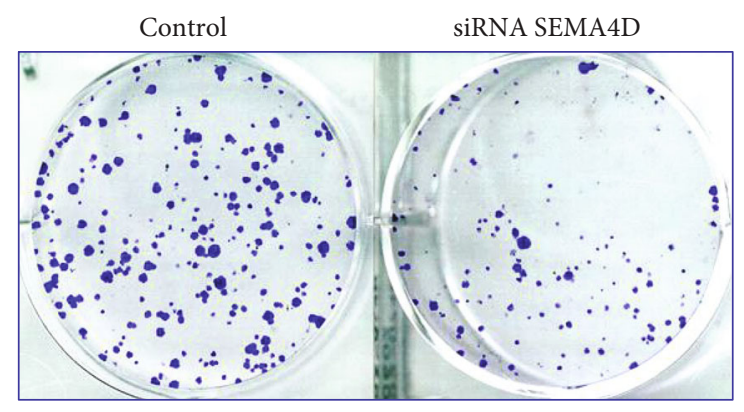

(c)

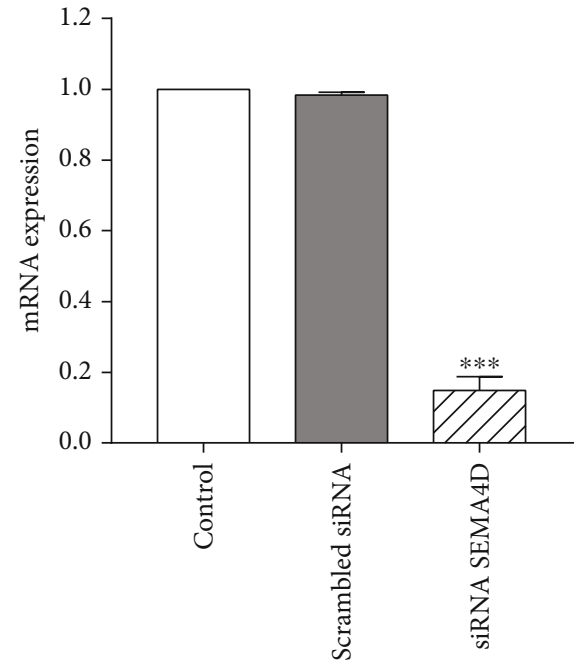

(b)
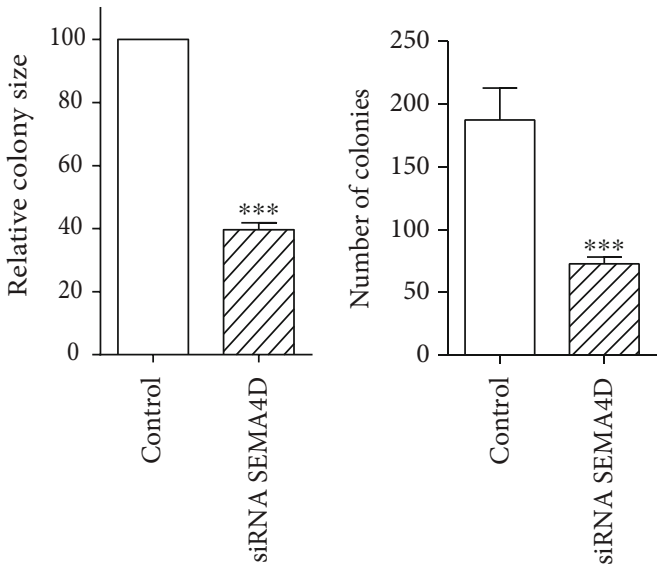

FIGURE 1: Effect of siRNA SEMA4D on SW48 cells viability. (a) MTT analysis of SW48 cells in the presence and absence of siRNA SEMA4D. (b) Cells were transfected with SEMA4D siRNA and scrambled siRNA and untransfected as control. RT-qPCR analysis revealed the downregulation of SEMA4D in siRNA SEMA4D transfected cells. (c) The effect of siRNA SEMA4D on the proliferation of SW48 cells was analyzed by colony formation assay. The findings are expressed as mean \pm standard deviation (SD) of three independent experiments. ${ }^{* * *} P<0.001$ compared with control.

compared by analysis of variance; $P<0.05$ was considered statistically significant.

\section{Results}

3.1. siRNA SEMA4D Suppressed SEMA4D Expression and Viability of SW48 Cells. SEMA4D siRNA was transfected into cells and the downregulation of SEMA4D was evaluated by RT-qPCR. As reported in an our previous study, SEMA4D siRNA caused markedly inhibition in the expression of SEMA4D in SW48 cells [25]. As it is also shown in Figure 1(b), there was no remarkable difference between the untransfected control and scrambled siRNA transfected cells for SEMA4D expression which confirmed the specific silencing effect of siRNA SEMA4D. Then, we performed MTT assay to investigate the influence of SEMA4D on cell viability and metabolic activity. As shown in Figure 1(a), the ratio of viable cells was decreased in the cells transfected by siRNA SEMA4D in comparison with control.

Since the ability to proliferate and generate colonies is a key feature of cancer cells, the colony forming capacity of transfected SW48 cells was investigated in this study. siRNA SEMA4D remarkably decreased the number and size of colonies relative to the control group (Figure 1(c)).

3.2. Downregulation of SEMA4D Induces Apoptosis in SW48 Cells. The effect of SEMA4D knockdown on apoptosis was assessed using Annexin V/PI assay. Flow cytometric analysis demonstrated that SEMA4D silencing increases the percentage of apoptotic cells compared to untransfected cells (Figure 2(a)). To check the effect of SEMA4D on the induction of apoptosis, we evaluated the expression of apoptosisrelated genes. mRNA expression levels of Caspase-3, P53, $\mathrm{Bcl} 2$, and Bax were thus analyzed. After SEMA4D depletion, 

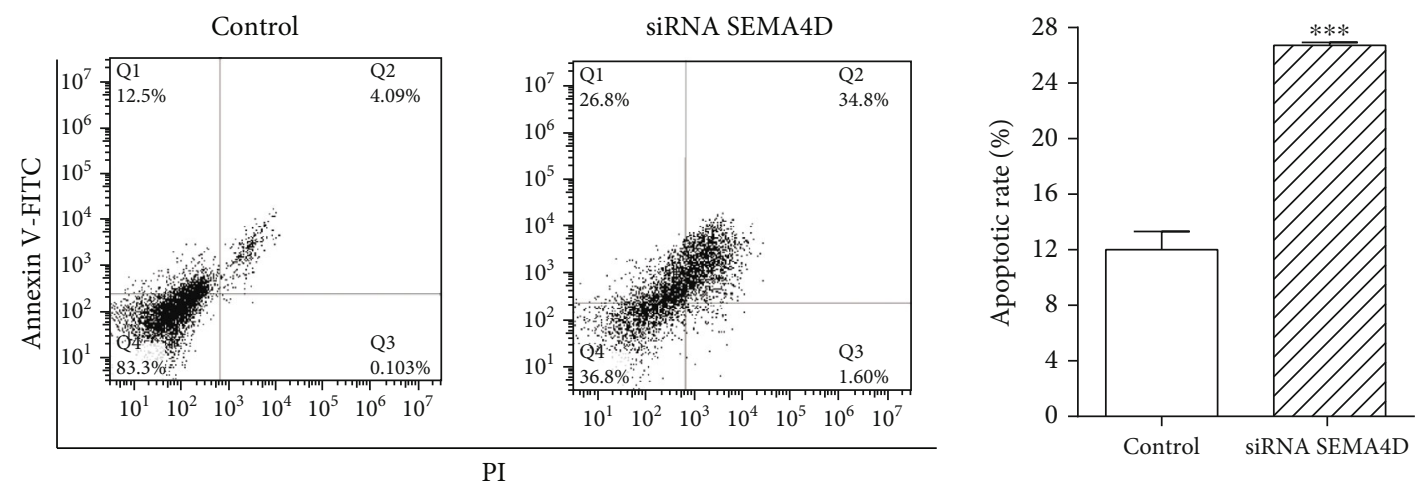

(a)

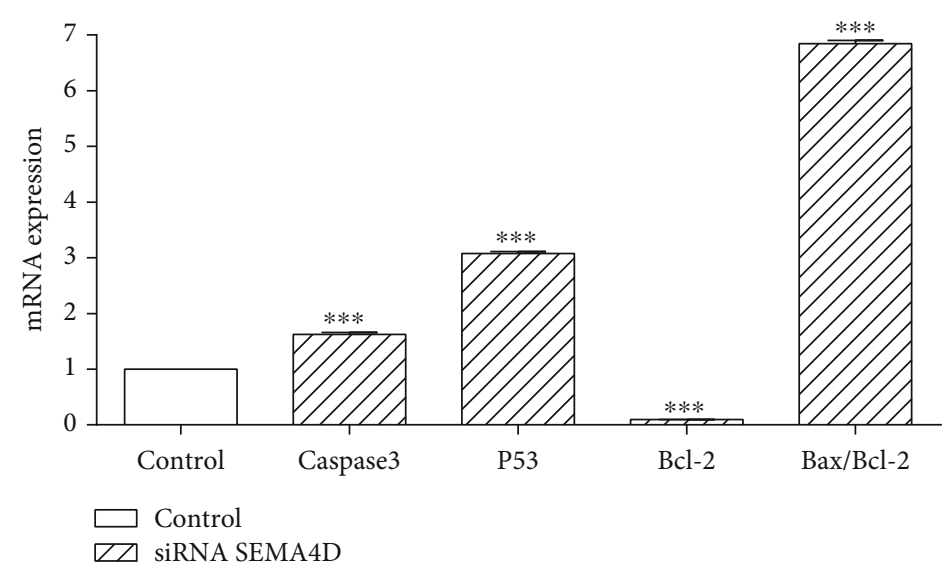

(b)

FIGURE 2: Effect of siRNA SEMA4D on SW48 cells apoptosis. (a) Induction of apoptosis was measured by flow cytometry analysis after $72 \mathrm{~h}$ in the presence and absence of siRNA SEMA4D. (b) mRNA levels of caspase 3, P53, Bcl2, and Bax were analyzed by qRT-PCR in transfected and untransfected SW48 cells. GAPDH was employed as an internal control. The data are expressed as mean \pm standard deviation, and each experiment was repeated three times. ${ }^{* * *} P<0.001$ vs. control group.

mRNA expression levels of Caspase-3, P53, as well as $\mathrm{Bax} / \mathrm{Bcl} 2$ ratio significantly increased, whereas there was a marked decrease in mRNA expression of Bcl-2 gene in SW48 cells transfected with siRNA SEMA4D in comparison with control group (Figure 2(b)).

\subsection{Knockdown of SEMA4D Suppressed CSCs and EMT} Markers via Downregulation of $\beta$-Catenin. As fundamental physiological processes, CSCs and EMT play critical roles in chemoresistance, invasion, tumor recurrence, and metastasis of tumor cells. To evaluate the effect of SEMA4D suppression on CSCs and EMT markers, SW48 cells were transfected with siRNA SEMA4D for $72 \mathrm{~h}$, and their mRNA expression was checked using RT-qPCR. Our findings revealed that transcript expression of CSC markers, including CD44, CD133, BMI1, ALDH1, and DCLK1, was significantly lower in transfected SW48 cells relative to untransfected cells (Figure 3(a)). A crucial feature of CSCs is their capacity to form spheres when growing in serumfree medium within nonadherent plates. To confirm the inhibition of CSC markers by siRNA SEMA4D, sphere formation assay was done to examine the ability of CSC self-renewal in SW48 cells. The findings indicated that the size and number of spheres in transfected SW48 cells was much lower after 7 days compared to control cells (Figure 3(b)).

Moreover, it was demonstrated that SEMA4D knockdown significantly increased the expression of E-cadherin as an epithelial marker, while mesenchymal markers such as $\mathrm{N}$-cadherin, Zeb1, and vimentin were remarkably reduced in comparison with control group (Figure 3(c)).

In addition, mRNA and protein expression levels of $\beta$ catenin as an oncogenic transcription factor were, respectively, analyzed by RT-qPCR and Western blot to illustrate the mechanism underlying the decrease in cancer stemness and EMT markers through silencing of SEMA4D. Our data revealed that mRNA and protein expression of $\beta$-catenin were strongly suppressed upon SEMA4D silencing (Figures 3(d) and $3(\mathrm{e})$ ). In addition, Western blot analysis was performed to confirm the gene expression results of E-cadherin. The findings indicated a considerable increase of E-cadherin protein expression in the presence of siRNA SEMA4D (Figure 3(e)).

3.4. SEMA4D Suppression Decreases SW48 Cells Migration. Scratch test was performed to detect the impact of SEMA4D silencing on migration ability of SW48 cells. Our findings exhibited that following transfection by siRNA, the scratches 


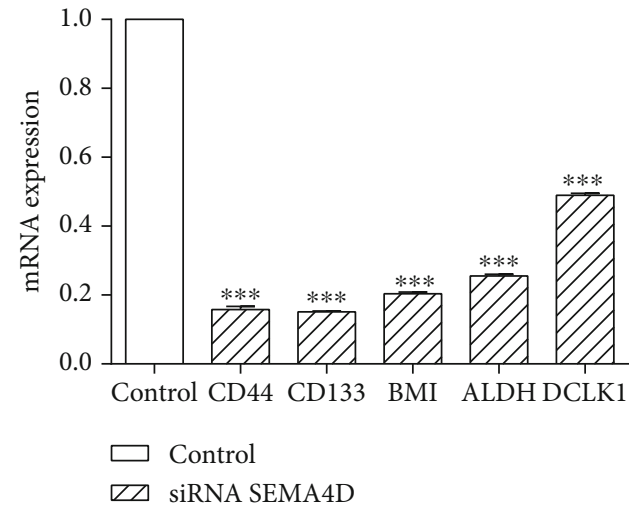

(a)

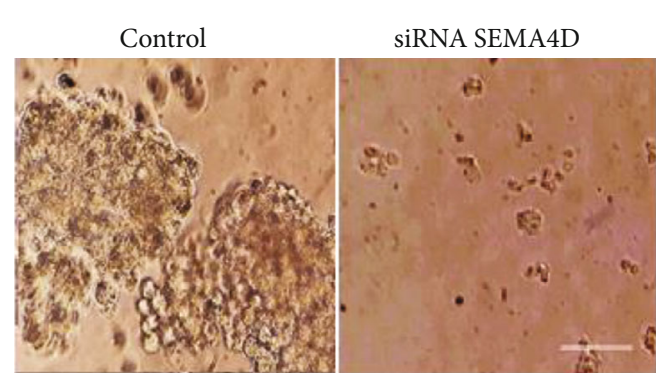

(b)

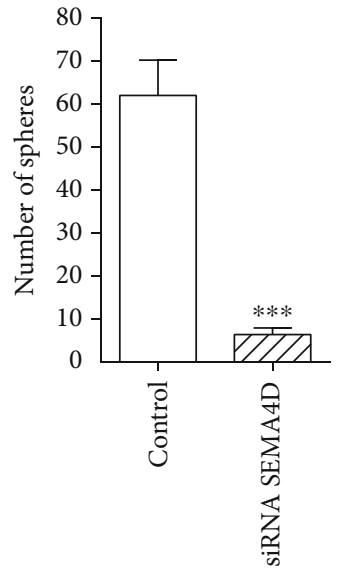

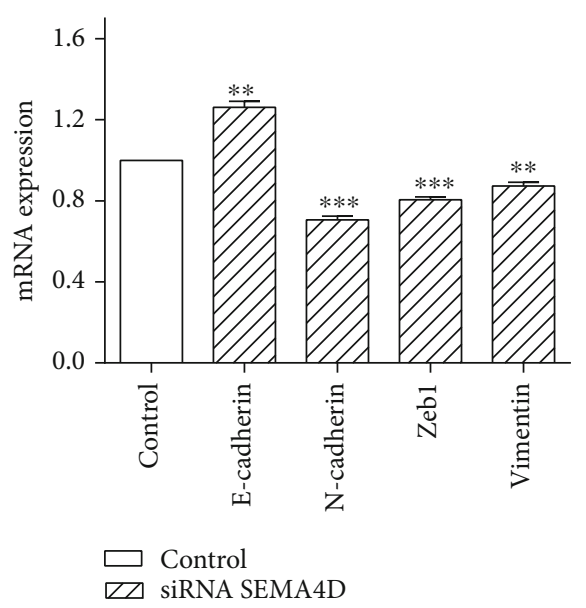

(c)

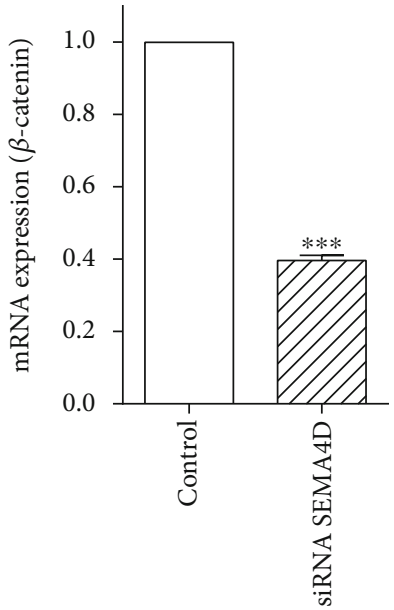

(d)

Figure 3: Continued. 

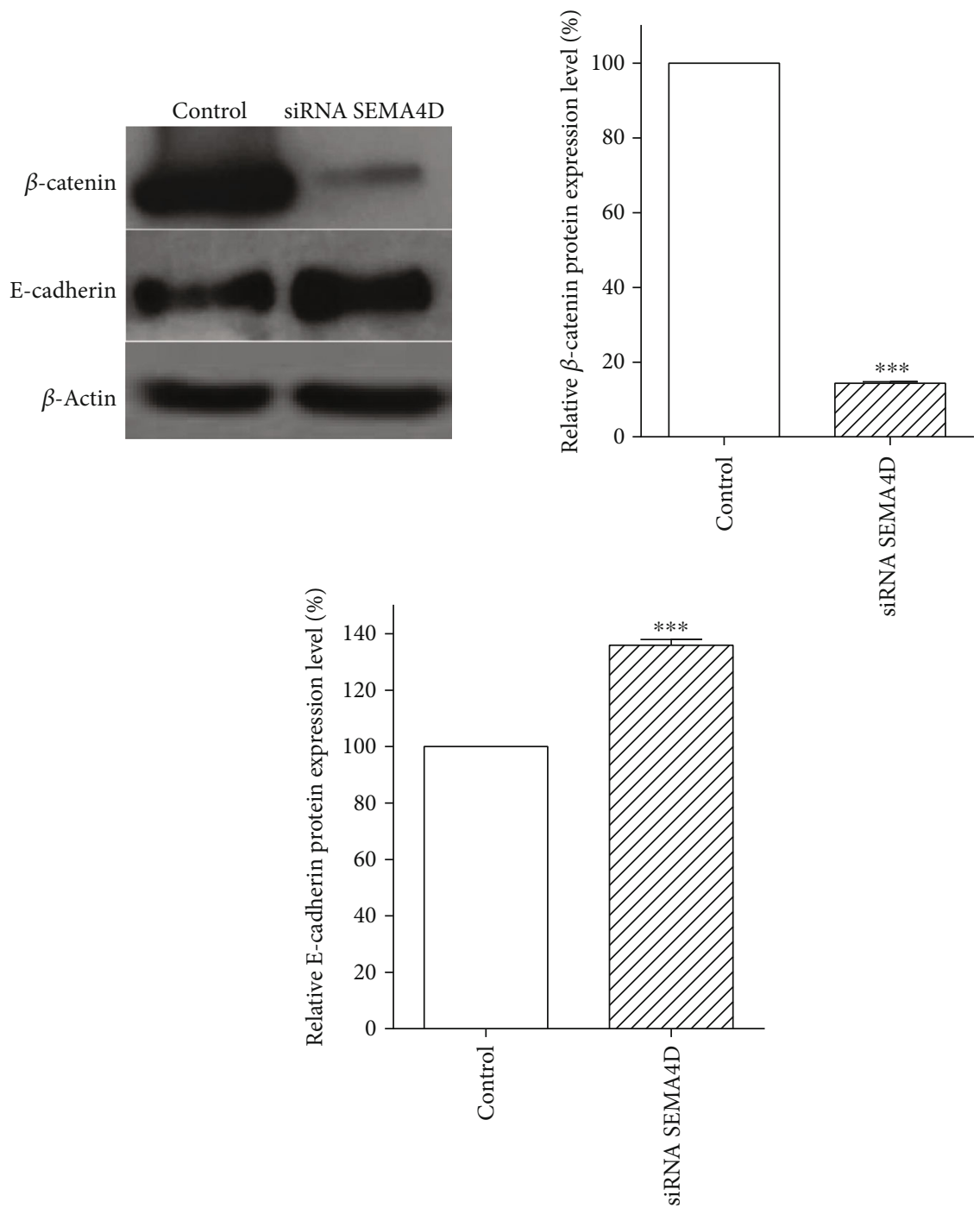

(e)

FIGURE 3: Effect of SEMA4D silencing on EMT- and CSC-related marker expression. (a) The mRNA expression levels of CSC-related genes were examined by RT-qPCR. (b) Representative images from sphere-forming assay. (c) RT-qPCR analysis of EMT-related markers. (d) Transcript abundance of $\beta$-catenin was analyzed by RT-qPCR. (e) $\beta$-catenin and E-cadherin protein expressions were determined by Western blot, and bar graph shows semiquantified densitometry from Western blot analysis. The data are expressed as mean \pm standard deviation, and each experiment was repeated three times. ${ }^{* * *} P<0.001$ vs. control group.

were wider than those of untransfected cells due to decreased number of cells migrating from the edge of the wound (Figure 4(a)). In addition, zymography was employed to measure hydrolytic activity of matrix metalloproteinase-2/9 (MMP-2/-9). The zymographic activity of MMP-2 was markedly decreased in transfected SW48 cells compared with untransfected cells. There was no considerable change in the activity of MMP-9 (Figure 4(b)). Real-time PCR analysis revealed that the expression of MMP-2 was remarkably diminished relative to control, and again, there was no significant difference in MMP-9 mRNA expression (Figure 4(c)).

\section{Discussion}

CRC remains one of the leading cause of human malignancies worldwide [29]. Although pioneering treatment strate- gies have been made, its morbidity and mortality are still substantial. Therefore, alternative strategies might be beneficial and effective. SEMA4D plays key roles in various mechanisms involved in cancer including tumor angiogenesis, cancer progression, metastasis, and also, tumor microenvironment regulation [30-32]. Notably, SEMA4D is overexpressed in various malignancies compared with normal tissue cells, such as prostate cancer [33], pancreatic cancer [18], cervical cancer [23], and particularly CRC [17, 24]. Detection of SEMA4D is correlated with a poor prognosis in CRC patients and can be used for predicting disease recurrence in CRC patients $[17,34]$.

It has been reported that lymphocytes infiltrated in the tumor stroma of pancreatic and colon cancer are responsible for SEMA4D expression $[17,18]$. However, several investigations reported that tumor-associated macrophages (TAMs) 

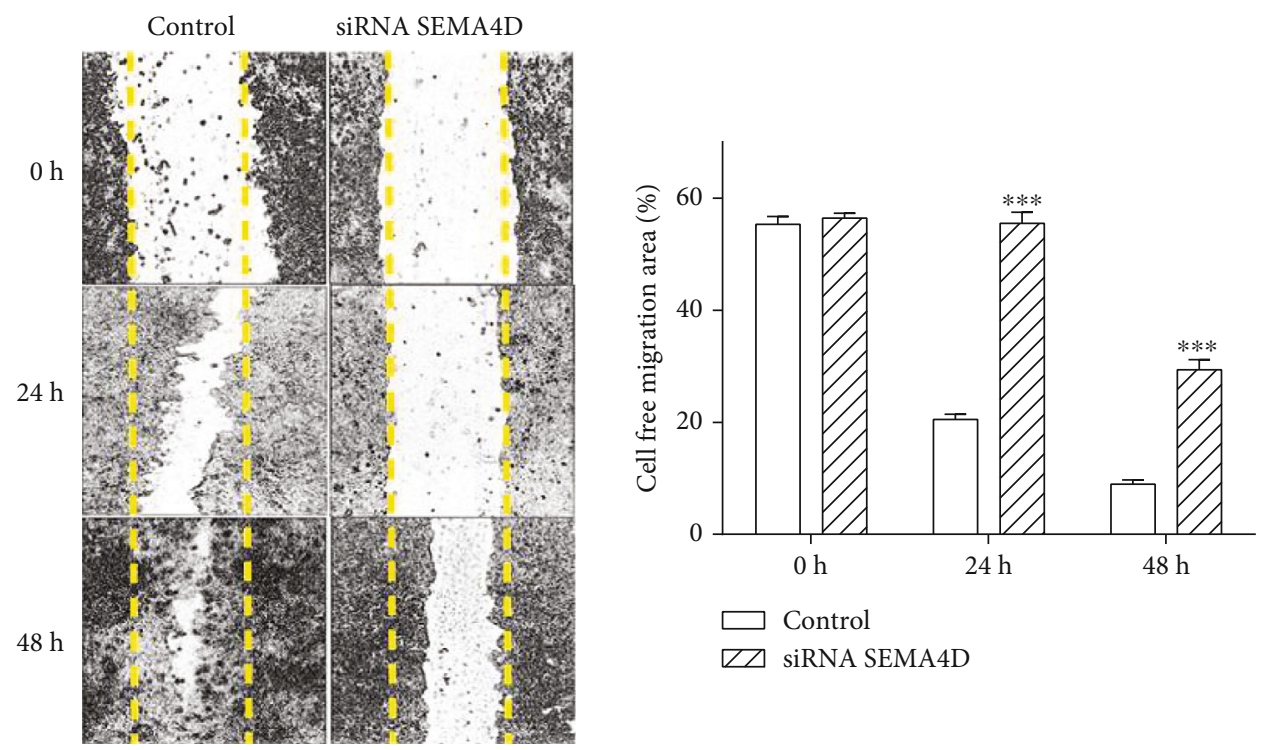

(a)
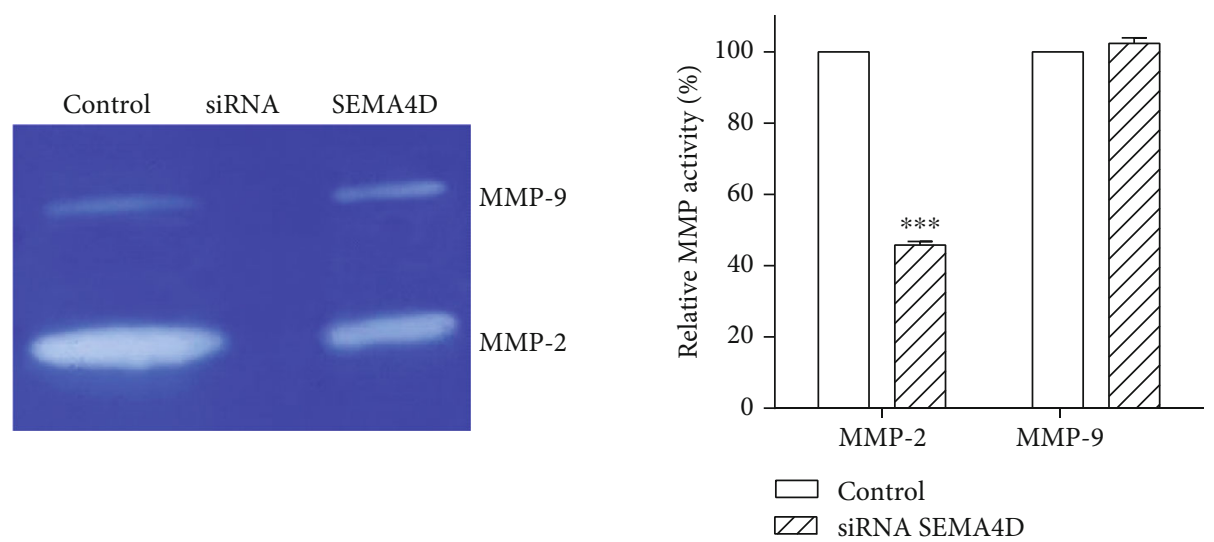

(b)

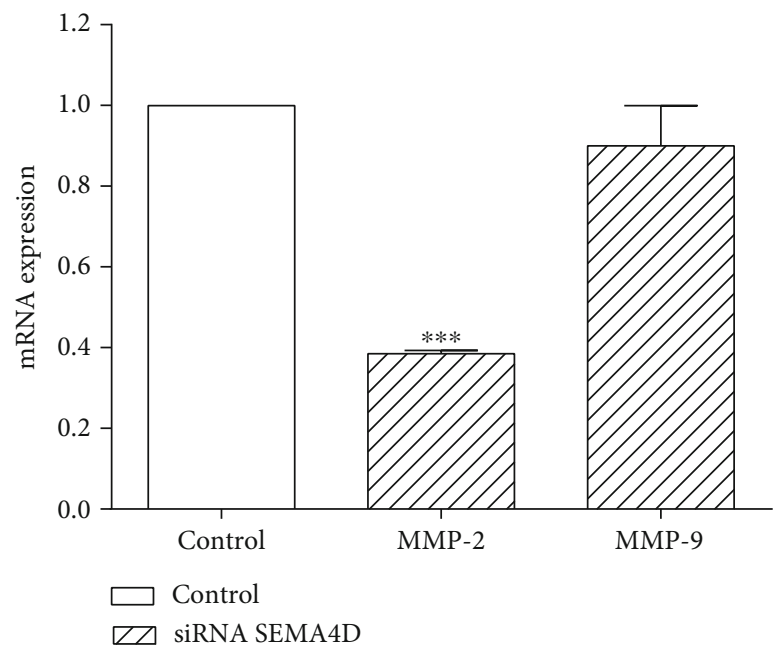

(c)

FIGURE 4: Effect of SEMA4D silencing on cell migration. (a) Representative image of SW48 cell migration in the absence and presence of siRNA SEMA4D at $0 \mathrm{~h}, 24 \mathrm{~h}$, and $48 \mathrm{~h}$. The scratched areas were measured in three random fields in each group. (b) The effect of siRNA SEMA4D on MMP-2 and MMP-9 activity, which was evaluated by gelatin zymography. Gelatinolytic activity of MMP-2 and MMP-9 is revealed in polyacrylamide gel by white banding. (c) The MMP-2 and MMP-9 gene expression was analyzed by real-time PCR. Data are represented as mean \pm standard deviation ${ }^{* * *} P<0.001$ vs. control group. 
considerably induced expression of SEMA4D in colon cells, and this overexpression is remarkably associated with lymphatic metastasis and specific histological types [24, 35]. A study conducted by Evans et al. illustrated an antitumor activity of SEMA4D blocking through antibody on the immune system [36]. Moreover, dihydromyricetin (DMY) exerted its antitumor effects through induction of the oxidative stress and inflammation in colon cancer cells following regulation of the SEMA4D expression [37].

While the expression abundance of SEMA4D has been investigated in the colorectal tissues, the correlation between silencing of SEMA4D expression and colon cell responses remains relatively unclear. Accordingly, the comprehension of new cellular and molecular mechanisms involved in tumor progression is pivotal for the development of novel therapeutic approaches. In our former study, it was showed that siRNA-mediated SEMA4D gene silencing can increase chemosensitivity to 5-FU in CRC through several oncogenic pathways particularly by promoting apoptosis [25], but in this study, we aimed to investigate whether SEMA4D inhibition can directly be effective in tumor suppression.

Hence, the purpose of this study was to demonstrate the changes in tumor progression mechanisms such as apoptosis, migration, EMT process, and $\beta$-catenin pathway followed by the silencing of SEMA4D expression using siRNA. This is, to our knowledge, the first study to investigate the relationship between knocking down of SEMA4D expression and $\beta$ catenin pathway-dependent EMT and CSCs in CRC.

In the present study, after the SEMA4D knockdown, the percentage of viable tumor cells decreased, and the rate of apoptosis increased. Moreover, SEMA4D silencing inhibited SW48 cell migration, the CSC markers, EMT process, $\beta$ catenin signaling pathway, colony formation, sphere formation, and MMP-2 activities.

CSCs are responsible for tumor initiation, tumor progression, and therapeutic resistance. Conventional CRC therapies are challengingly failed to target CSCs because of their extreme persistence to the treatment; consequently, they may be causative of tumor recurrence and metastasis [38]. The present observations support considerable downregulation of CSC markers such as CD44, CD133, ALDH, DCLK1, and BMI1 as well as inhibition of sphere-forming assay as an in vitro assay for the determination and enrichment of CSCs. Interestingly, CSCs can participate in TAMs infiltration and polarization in tumor stroma [39]. Therefore, SEMA4D silencing not only might directly lead to a decrease of CSCs but also, it might indirectly contribute to the suppression of TAMs recruiting. Moreover, TAMs implicate tumor progression through EMT and angiogenesis. Subsequently, acquiring an EMT phenotype leads to cancer cell invasion and tumor metastasis.

Several lines of evidence have illustrated that CRC cells with an EMT phenotype are rich sources for CSCs, indicating a biological link between EMT and CSCs [38]. Here, after inhibition of SEMA4D, the expression levels of EMT markers including mesenchymal markers such as $\mathrm{N}$-cadherin and vimentin as well as EMT transcription factor (Zeb1) were diminished compared with untreated cells; however, Ecadherin as an epithelial marker was elevated. E-cadherin plays an underlying role in the inhibition of invasion, and its regulation might result in repression of tumor progression (15).

Activation of EMT is induced through a prominent selfrenewal pathway: $\mathrm{WNT} / \beta$-catenin. It is demonstrated that overactivation of the WNT/ $\beta$-catenin pathway could repress E-cadherin and promote EMT process and local invasion in the colorectal tumor $[40,41]$. Our data revealed that the mRNA expression of $\beta$-catenin and its protein level downregulated following transfection of the siRNA SEMA4D. Therefore, $\beta$-catenin oncogene inhibition might be effective on enhanced E-cadherin and suppressed EMT and CSC markers. Indeed, it has been demonstrated that $\beta$-catenin suppression which is considered as a growth stimulus in a subset of colon cancer cell lines led to tumor regression [42, 43]. Furthermore, it has been reported that the depletion of E-cadherin contributes to induce EMT, eventually leading to migration [44].

Another critical factor for tumor progression is migration via the ECM that involves several types of molecules, including MMPs and chemokine [45]. MMPs are expressed in different stages of CRC and linked with survival and prognosis [46]. In this article, we concentrated on MMP-2 and MMP9 , due to their high expression reporting by various cancers investigations including CRC [46, 47].

SEMA4D knockdown significantly repressed cell migration ability and mRNA expression of MMP-2 as well as its enzymatic activity, whereas the mRNA expression and enzymatic activity of MMP-9 stayed constant after transfection of siRNA SEMA4D. It is revealed that the cancer cells could promote invasion in an MMP2-dependent manner following E-cadherin knockdown [48] As reported by Yan Wang et al., SEMA4D expression has been reported to be increased in esophagus squamous cell carcinoma (ESCC), and consistent with our results, SEMA4D knockdown could repress cell proliferation and migration in ESCC [49].

Several studies in the course of recent years have indicated the significant role of apoptosis in various malignancies and its promising role in anticancer therapy [50]. Defect in apoptosis is a hallmark of human cancer and leads to an aggressive tumor phenotype correlating with drug resistance and failure of treatments including chemotherapy, radiation, and targeted therapies [51].

Apoptotic signaling pathways which are crucial for tumor survival deregulated in CRC, and this dysregulation mainly correlated with overexpression of $\mathrm{Bcl}-2$ family proteins [52]. Evasion of apoptosis and the disproportionateness between proapoptotic and antiapoptotic protein levels are critical factors in the pathogenesis of cancer [53]. In addition, the apoptosis evasion of CSCs is considered as a prominent element for the failure of existing therapies to abolish tumors [52].

As predicted, our results demonstrate that SEMA4D knockdown markedly accelerated the rate of apoptotic cells compared with nontransfected cells. It was also associated with the reduced expression of $\mathrm{Bcl}-2$ and the elevated expression of P53, caspase-3, and Bax/Bcl-2 ratio. Management of the apoptotic pathways, including deactivation of antiapoptotic pathways and stimulation of proapoptotic markers to eliminate tumor cells, demonstrates significant potentials. 
In line with our findings, it has been indicated that SEMA4D silencing elevated the apoptosis rate of KYSE-150 and TE-10 cells [49]. In addition, the upregulation of SEMA4D in HP45 cells could suppress the apoptosis, and SEMA4D targeting by miR-125b could negatively regulate it and augment the apoptosis rate in avian leucosis virus-transformed cells [54]. As reported by Liu et al., it has been revealed that targeting of SEMA4D by miR-214 could prevent cell proliferation and induce apoptosis in ovarian cancer cells [55]. The findings from the present study indicate that SEMA4D knockdown by siRNA in human colorectal cancer cells suppressed various oncogenic pathways, including inhibition of $\beta$-catenin pathway-dependent EMT and CSCs, attenuating cell growth and invasion through diminution MMP-2 enzymatic activity and apoptosis augmentation. It is possible that SEMA4D silencing may prevent TAM infiltration and polarization in tumor stroma which is potentially required for SEMA4D production. Further investigation will be necessary to elucidate the mechanism of suppressive effect of SEMA4D silencing on the recruiting of TAMs. Ultimately, this data suggest that SEMA4D might be considered a substantial cornerstone for the development of CRC-specific therapeutics.

\section{Data Availability}

The data that support the findings of this study are available in the manuscript.

\section{Conflicts of Interest}

The authors declare that they have no conflict of interest.

\section{Authors' Contributions}

Mahsa Rezaeepoor and Golnaz Rashidi contributed equally to this manuscript.

\section{Acknowledgments}

This study was supported by a grant from the Hamadan University of Medical Sciences, Hamadan, Iran (No. 9705162835).

\section{Supplementary Materials}

Uncropped Western blots corresponding to Figure 3(e). (Supplementary Materials)

\section{References}

[1] A. J. Cornish, P. J. Law, M. Timofeeva et al., "Modifiable pathways for colorectal cancer: a mendelian randomisation analysis," The Lancet Gastroenterology \& Hepatology, vol. 5, no. 1, pp. 55-62, 2020.

[2] S.-W. Pang, N. J. Awi, S. Armon et al., "Current update of laboratory molecular diagnostics advancement in management of colorectal cancer (CRC)," Diagnostics, vol. 10, no. 1, p. 9, 2020.

[3] E. Koncina, S. Haan, S. Rauh, and E. Letellier, "Prognostic and predictive molecular biomarkers for colorectal cancer: updates and challenges," Cancers, vol. 12, no. 2, p. 319, 2020.
[4] F. Karimi Dermani, R. Amini, M. Saidijam, and R. Najafi, "miR-200c, a tumor suppressor that modulate the expression of cancer stem cells markers and epithelial-mesenchymal transition in colorectal cancer," Journal of Cellular Biochemistry, vol. 119, no. 7, pp. 6288-6295, 2018.

[5] F. Papaccio, F. Paino, T. Regad, G. Papaccio, V. Desiderio, and V. Tirino, "Concise review: cancer cells, cancer stem cells, and mesenchymal stem cells: influence in cancer development," Stem Cells Translational Medicine, vol. 6, no. 12, pp. 21152125, 2017.

[6] T. Brabletz, A. Jung, S. Spaderna, F. Hlubek, and T. Kirchner, "Migrating cancer stem cells - an integrated concept of malignant tumour progression," Nature Reviews Cancer, vol. 5, no. 9, pp. 744-749, 2005.

[7] A. Kabashima-Niibe, H. Higuchi, H. Takaishi et al., "Mesenchymal stem cells regulate epithelial-mesenchymal transition and tumor progression of pancreatic cancer cells," Cancer Science, vol. 104, no. 2, pp. 157-164, 2013.

[8] P. Lindner, S. Paul, M. Eckstein et al., "EMT transcription factor ZEB1 alters the epigenetic landscape of colorectal cancer cells," Cell Death \& Disease, vol. 11, no. 2, pp. 1-13, 2020.

[9] V. H. L. Nguyen, R. Hough, S. Bernaudo, and C. Peng, "Wnt/ $\beta$-catenin signalling in ovarian cancer: insights into its hyperactivation and function in tumorigenesis," Journal of Ovarian Research, vol. 12, no. 1, p. 122, 2019.

[10] J. Huang, D. Xiao, G. Li et al., "EphA2 promotes epithelialmesenchymal transition through the $\mathrm{Wnt} / \beta$-catenin pathway in gastric cancer cells," Oncogene, vol. 33, no. 21, pp. 27372747, 2014.

[11] A. Sebio, M. Kahn, and H.-J. Lenz, The Potential of Targeting Wnt/ $\beta$-Catenin in Colon Cancer, Taylor \& Francis, 2014.

[12] S. E. Baldus, S. P. Mönig, S. Huxel et al., "MUC1 and nuclear $\beta$ catenin are coexpressed at the invasion front of colorectal carcinomas and are both correlated with tumor prognosis," Clinical Cancer Research, vol. 10, no. 8, pp. 2790-2796, 2004.

[13] T. Brabletz, F. Hlubek, S. Spaderna et al., "Invasion and metastasis in colorectal cancer: epithelial-mesenchymal transition, mesenchymal-epithelial transition, stem cells and $\beta$-catenin," Cells, Tissues, Organs, vol. 179, no. 1-2, pp. 56-65, 2005.

[14] G. Neufeld, Y. Mumblat, T. Smolkin et al., "The role of the semaphorins in cancer," Cell Adhesion \& Migration, vol. 10, no. 6, pp. 652-674, 2016.

[15] L. Tamagnone and G. Franzolin, "Targeting semaphorin 4D in cancer: a look from different perspectives," Cancer Research, vol. 79, no. 20, pp. 5146-5148, 2019.

[16] J. R. Basile, A. Barac, T. Zhu, K. L. Guan, and J. S. Gutkind, "Class IV semaphorins promote angiogenesis by stimulating rho-initiated pathways through plexin-B," Cancer Research, vol. 64, no. 15, pp. 5212-5224, 2004.

[17] T. Ikeya, K. Maeda, H. Nagahara, M. Shibutani, Y. Iseki, and K. Hirakawa, "The combined expression of Semaphorin4D and PlexinB1 predicts disease recurrence in colorectal cancer," BMC Cancer, vol. 16, no. 1, p. ???, 2016.

[18] S. Kato, K. Kubota, T. Shimamura et al., "Semaphorin 4D, a lymphocyte semaphorin, enhances tumor cell motility through binding its receptor, plexinB1, in pancreatic cancer," Cancer Science, vol. 102, no. 11, pp. 2029-2037, 2011.

[19] T. Worzfeld, J. M. Swiercz, M. Looso, B. K. Straub, K. K. Sivaraj, and S. Offermanns, "ErbB-2 signals through PlexinB1 to promote breast cancer metastasis," The Journal of Clinical Investigation, vol. 122, no. 4, pp. 1296-1305, 2012. 
[20] Y. Chen, L. Zhang, R. Lv, and W. Q. Zhang, "Overexpression of Semaphorin $4 \mathrm{D}$ indicates poor prognosis and prompts monocyte differentiation toward M2 macrophages in epithelial ovarian cancer," Asian Pacific Journal of Cancer Prevention, vol. 14, no. 10, pp. 5883-5890, 2013.

[21] M. F. A. Malik, L. Ye, and W. G. Jiang, "Reduced expression of semaphorin $4 \mathrm{D}$ and plexin-B in breast cancer is associated with poorer prognosis and the potential linkage with oestrogen receptor," Oncology Reports, vol. 34, no. 2, pp. 1049-1057, 2015.

[22] Y. Chen, L. Zhang, Y. Pan, X. Ren, and Q. Hao, "Over-expression of semaphorin $4 \mathrm{D}$, hypoxia-inducible factor- $1 \alpha$ and vascular endothelial growth factor is related to poor prognosis in ovarian epithelial cancer," International Journal of Molecular Sciences, vol. 13, no. 12, pp. 13264-13274, 2012.

[23] H. Liu, Y. Yang, J. Xiao et al., "Semaphorin 4D expression is associated with a poor clinical outcome in cervical cancer patients," Microvascular Research, vol. 93, pp. 1-8, 2014.

[24] L. Mu, J. Wang, X. Guo et al., "Correlation and clinical significance of expressions of HIF- $1 \alpha$ and Sema4D in colorectal carcinoma tissues," Zhonghua wei chang wai ke za zhi= Chinese Journal of Gastrointestinal Surgery, vol. 17, no. 4, pp. 388392, 2014.

[25] G. Rashidi, M. Rezaeepoor, C. Mohammadi, G. Solgi, and R. Najafi, "Inhibition of semaphorin 4D enhances chemosensitivity by increasing 5 -fluorouracile-induced apoptosis in colorectal cancer cells," Molecular Biology Reports, vol. 47, no. 9, pp. 7017-7027, 2020.

[26] N. A. Franken, H. M. Rodermond, J. Stap, J. Haveman, and C. van Bree, "Clonogenic assay of cells in vitro," Nature Protocols, vol. 1, no. 5, pp. 2315-2319, 2006.

[27] L. Lan, Y. Luo, D. Cui et al., "Epithelial-mesenchymal transition triggers cancer stem cell generation in human thyroid cancer cells," International Journal of Oncology, vol. 43, no. 1, pp. 113-120, 2013.

[28] R. Roudi, Z. Madjd, M. Ebrahimi et al., "Evidence for embryonic stem-like signature and epithelial-mesenchymal transition features in the spheroid cells derived from lung adenocarcinoma," Tumor Biology, vol. 37, no. 9, pp. 1184311859, 2016.

[29] M. Karimi Mazraehshah, S. M. Tavangar, M. Saidijam et al., "Anticancer effects of miR-200c in colorectal cancer through BMI1," Journal of Cellular Biochemistry, vol. 119, no. 12, pp. 10005-10012, 2018.

[30] H. Zhou, N. O. Binmadi, Y.-H. Yang, P. Proia, and J. R. Basile, "Semaphorin 4D cooperates with VEGF to promote angiogenesis and tumor progression," Angiogenesis, vol. 15, no. 3, pp. 391-407, 2012.

[31] G. Valente, G. Nicotra, M. Arrondini et al., "Co-expression of plexin-B1 and Met in human breast and ovary tumours enhances the risk of progression," Analytical Cellular Pathology., vol. 31, no. 6, pp. 423-436, 2009.

[32] S. Giordano, S. Corso, P. Conrotto et al., "The semaphorin 4D receptor controls invasive growth by coupling with Met," Nature cell biology., vol. 4, no. 9, pp. 720-724, 2002.

[33] R. W. Ross, M. D. Galsky, H. I. Scher et al., "A whole-blood RNA transcript-based prognostic model in men with castration- resistant prostate cancer: a prospective study," The lancet oncology., vol. 13, no. 11, pp. 1105-1113, 2012.

[34] J.-S. Wang, C.-Q. Jing, K.-S. Shan et al., "Semaphorin 4D and hypoxia-inducible factor- $1 \alpha$ overexpression is related to prog- nosis in colorectal carcinoma," World Journal of Gastroenterology: WJG, vol. 21, no. 7, pp. 2191-2198, 2015.

[35] J. R. Sierra, S. Corso, L. Caione et al., "Tumor angiogenesis and progression are enhanced by Sema4D produced by tumorassociated macrophages," The Journal of Experimental Medicine, vol. 205, no. 7, pp. 1673-1685, 2008.

[36] E. E. Evans, A. S. Jonason, H. Bussler et al., “Antibody blockade of semaphorin $4 \mathrm{D}$ promotes immune infiltration into tumor and enhances response to other immunomodulatory therapies," Cancer Immunology Research, vol. 3, no. 6, pp. 689701, 2015.

[37] J. Liang, J. Wu, F. Wang, P. Zhang, and X. Zhang, "Semaphoring $4 \mathrm{D}$ is required for the induction of antioxidant stress and anti- inflammatory effects of dihydromyricetin in colon cancer," International Immunopharmacology, vol. 67, pp. 220230, 2019.

[38] A. Z. Ayob and T. S. Ramasamy, "Cancer stem cells as key drivers of tumour progression," Journal of Biomedical Science, vol. 25, no. 1, p. 20, 2018.

[39] N.-B. Hao, M.-H. Lü, Y.-H. Fan, Y. L. Cao, Z. R. Zhang, and S. M. Yang, "Macrophages in tumor microenvironments and the progression of tumors," Clinical and Developmental Immunology, vol. 2012, 11 pages, 2012.

[40] T. Vu and P. K. Datta, "Regulation of EMT in colorectal cancer: a culprit in metastasis," Cancers, vol. 9, no. 12, p. 171, 2017.

[41] J. I. Yook, X.-Y. Li, I. Ota, E. R. Fearon, and S. J. Weiss, "Wntdependent regulation of the E-cadherin repressor snail," Journal of Biological Chemistry, vol. 280, no. 12, pp. 11740-11748, 2005.

[42] J.-S. Kim, H. Crooks, A. Foxworth, and T. Waldman, "Proofof-principle: oncogenic beta-catenin is a valid molecular target for the development of pharmacological inhibitors," Molecular Cancer Therapeutics, vol. 1, no. 14, pp. 1355-1359, 2002.

[43] R. Thakur and D. P. Mishra, "Pharmacological modulation of beta-catenin and its applications in cancer therapy," Journal of Cellular and Molecular Medicine, vol. 17, no. 4, pp. 449-456, 2013.

[44] J. P. Thiery, "Epithelial-mesenchymal transitions in tumour progression," Nature Reviews Cancer, vol. 2, no. 6, pp. 442454, 2002.

[45] M. Pourjafar, M. Saidijam, K. Etemadi, and R. Najafi, “Alltrans retinoic acid enhances in vitro mesenchymal stem cells migration by targeting matrix metalloproteinases 2 and 9," Biotechnology Letters, vol. 39, no. 8, pp. 1263-1268, 2017.

[46] R. A. Wagenaar-Miller, L. Gorden, and L. M. Matrisian, "Matrix metalloproteinases in colorectal cancer: is it worth talking about?," Cancer and Metastasis Reviews, vol. 23, no. 1/2, pp. 119-135, 2004.

[47] A. Morán, P. Iniesta, C. García-Aranda et al., "Clinical relevance of MMP-9, MMP-2, TIMP-1 and TIMP-2 in colorectal cancer," Oncology Reports, vol. 13, no. 1, pp. 115120, 2005.

[48] G.-Y. Bae, S.-J. Choi, J.-S. Lee et al., "Loss of E-cadherin activates EGFR-MEK/ERK signaling, which promotes invasion via the ZEB1/MMP2 axis in non-small cell lung cancer," Oncotarget, vol. 4, no. 12, pp. 2512-2522, 2013.

[49] Y. Wang, H. Zhao, and W. Zhi, "SEMA4D under the posttranscriptional regulation of HuR and miR-4319 boosts cancer progression in esophageal squamous cell carcinoma," Cancer Biology \& Therapy, vol. 21, no. 2, pp. 122-129, 2020. 
[50] C. Pfeffer and A. Singh, "Apoptosis: a target for anticancer therapy," International Journal of Molecular Sciences, vol. 19, no. 2, p. 448, 2018.

[51] L. Zhang and J. Yu, "Role of apoptosis in colon cancer biology, therapy, and prevention," Current Colorectal Cancer Reports, vol. 9, no. 4, pp. 331-340, 2013.

[52] Y.-C. He, F.-L. Zhou, Y. Shen, D. F. Liao, and D. Cao, “Apoptotic death of cancer stem cells for cancer therapy," International Journal of Molecular Sciences, vol. 15, no. 5, pp. 83358351, 2014.

[53] T. Knight, D. Luedtke, H. Edwards, J. W. Taub, and Y. Ge, “A delicate balance - the BCL-2 family and its role in apoptosis, oncogenesis, and cancer therapeutics," Biochemical Pharmacology, vol. 162, pp. 250-261, 2019.

[54] C. Ren, R. Xie, Y. Yao et al., "MiR-125b suppression inhibits apoptosis and negatively regulates sema4D in avian leukosis virus-transformed cells," Viruses, vol. 11, no. 8, p. 728, 2019.

[55] Y. Liu, H. Zhou, L. Ma et al., "MiR-214 suppressed ovarian cancer and negatively regulated semaphorin 4D," Tumor Biology, vol. 37, no. 6, pp. 8239-8248, 2016. 